\title{
0883 THE DANGER OF POOR VISIBILITY - THE NIGERIAN EXPERIENCE
}

P Adenusi* Correspondence: El-Elyon (VALU4LIFE), 49 Bamgbose Street, Lagos Island, P.O. Box 73436 Victoria Island, Lagos NG234, Nigeria

10.1136/ip.2010.029215.883

In the developing countries lots can be achieved in Road Safety through Public Private Partnerships (PPP). Between 1992 and 2002 records shows that night time crashes contributed hugely to the number of road casualties. Research showed most crashes were related to rear impact because of poor visibility of stalled Heavy Goods Vehicles. The high death rate led to a public outcry which led to a national policy on the compulsory use of retro-reflective tape by 2004. The use of retro-reflective tape led to a high reduction in our national casualty record. There was reduction in the destruction of goods and properties; Less death of bread winners; Less burden for the Federal Road Safety Commission (FRSC) personnel. El-Elyon, a private company pioneered the use of the tape, while the FRSC enforced the provision of the law concerning the use of retro-reflective tape. El-Elyon engaged in advocacy for about 18 months and the campaign was nationwide with about 40\% compliance. P. Olson/M. Sivak researched in 1988 that the amount of light required to see during the hours of darkness doubles every 13 years. As we age, our visual capacity diminishes. The place of enhanced visibility cannot be over emphasised in the developing countries where they have poor maintenance culture, search \& rescue and poor medical services. The Nigerian experience led to about $30 \%$ reduction. This partnership is recommended for other developing countries with similar challenges. Transporters bear the cost of the tape. 